Journal of Engineering and Applied Sciences 14 (3): 920-926, 2019

ISSN: 1816-949X

(C) Medwell Journals, 2019

\title{
Fuzzy Logic Decision Fusion in a Fingerprints Based Multimodal Biometric System
}

\author{
Emad Majeed Hameed, Noor Abbood and Ahmed A. Alani \\ Baquba Technical Institute, Middle Technical University, Baquba, Iraq
}

\begin{abstract}
Multimodal biometric systems use more than one biometric trait or more than one algorithm for estimating the similarity. These systems allow using more factors for making decision. Since, the classical binary logic and threshold comparison do not give an ideal results in making decision, fuzzy logic is one approaches for solving this problem. This study presents a multimodal biometric system that uses three different fingerprints with fuzzy fusion system for making decision. The EER of fuzzy fusion based multimodal system is calculated and compared with other two fusion methods (majority voting and a response of three out three). Experimental results explain an improvement of about 10 and $12 \%$ using the fuzzy logic decision fusion over the fusion by majority voting and with a response of three out three, respectively.
\end{abstract}

Key words: Biometric, multimodal, fingerprint, biometric fusion, fuzzy logic fusion, voting

\section{INTRODUCTION}

Now a days information technology has penetrated almost all fields of life. The security problem of access to information systems and private information has become extremely relevant. Classical user authentication depends on passwords and keys can be easily lost or forgotten. Also, it does not guarantee that the password is used by the person whose access authentication. This problem can be solved by using biometric authentication, i.e., Authentication which checks the identity of the user based on his physiological or behavioral characteristics, such as facial features, voice, fingerprints.

The performance in biometric authentication is often affected by external conditions and variability. The data obtained from the sensors depend on the noise caused by the environment and the physiological characteristics of the person (sensor purity, illumination, body temperature, human age) (Jain et al., 1999; Brunelli and Falavigna, 1995).This leads to a fact that the decision on the identity of persons has a probabilistic nature. Thus, most of the researches in the field of biometrics aim to increase the accuracy and reliability of systems. The most systems frequently used to improve the quality of making decision are multimodal biometric systems. These systems use more than one biometric feature or more than one algorithm for estimating the similarity.

The multimodal biometric systems allow using more factors for making decision. Because of the complexity of these systems, the classical binary logic and threshold comparison do not give an ideal result. Many fusion techniques of multimodal biometric systems used in previous research are majority voting, sum or product rules, different classifier types like SVM, Bayesian classifier, decision trees and k-NN (Kittler et al., 1998; Jin et al., 2003).

The decision making based on fuzzy logic is one of the approaches for solving this problem. Fuzzy logic is a branch of mathematics that is a generalization of classical logic and set theory. The output in the fuzzy logic is based on variables that are not defined as binary membership but as continuous membership. This research proposes a multimodal biometric system that uses three different fingerprints with fuzzy fusion system for making decision. A comparison of this fuzzy logic fusion system is done with other simple techniques such as fusion by majority voting or response of three out three.

Literature review: A number of works have been conducted on multimodal biometrics and these studies show that multi biometric has more ideal results than single- biometric. Brunelli and Falavigna (1995) described a multimodal biometric system for fingerprint recognition using the index and middle fingers. The quality of fingerprnt was used for making a decision about identity.

Lau et al. (2004) presented a multimodal biometric system that uses voice recording, face photography and fingerprint. They used fuzzy logic decision fusion in order to consider the external conditions that affect verification, such as finger placement, pressure, sweat in fingerprint verification, lightning conditions and head positioning in face identification.

Vasuhi et al. (2010) developed system depending on fuzzy logic decision fusion of fingerprint and voice. The

Corresponding Author: Emad Majeed Hameed, Baquba Technical Institute, Middle Technical University, Baquba, Iraq 
work succeeded to overcome the defects of individual sensors. The experiments explain that the suggested way for fuzzy logic authentication is efficient.

Abdolahi et al. (2013) proposed a multi-modal biometric system (Fingerprint and Iris) with fuzzy logic decision level fusion to combine the results. The use of fuzzy logic gave flexible result with an additional improvement of $1.7 \%$.

Sharma and Singh (2017) used two uni-modal biometrics; fingerprint and face as multi-biometrics. Using fuzzy fusion of fingerprint and face recognition gives high accuracy compared with other fusion methods. In this study, we developed a multimodal biometric system using three fingerprints with fuzzy logic decision fusion. The quality of the fingerprnt image was evaluated by several different algorithms including the standard NFIQ, background selection and evaluating the brightness. The fuzzy fusion is conducted at decision level.

\section{MATERIALS AND METHODS}

Multimodal biometric systems: Biometric systems are divided into two classes; monomodal and multimodal. The standard monomodal system is constructed as in (Fig. 1).

A multimodal biometric recognition system can be carried out depending on evidences acquired of the multiple sources of biometric information. Multi biometric systems can be categorized considering the number of traits, sensors and feature sets used. a variety categories of multimodal systems can be distinguished (Ross and Jain, 2004; Poh et al., 2002; Prabhakar and Jain, 2002):

Single biometric trait, multiple sensors: In these systems, multiple sensors are used to capture the same biometric trait. Thus, raw biometric data relating to different sensors are obtained. Chang et al. acquired both
2 and 3D images of the face and integrate them at the data level as well as the match score level for getting improvement in the performance of a face recognition system.

Single biometric trait, multiple algorithms: Unlike the previous scenario, multi-algorithm systems employ only a single sensor to obtain raw data; this data is then used by two or more different matching algorithms. Ross et al. (2003) used two different matching algorithms in their fingerprint recognition system. They combine the matching score of a minutiae-based fingerprint matcher with that of a texture-based matcher to improve matching performance.

Single biometric trait, multiple units: Multiple units (Multi-instance) systems include combining information presented from multiple instances within the same biometric trait. In the case of fingerprints or iris, it is possible to integrate information obtained by 2 or more fingers (or both the irises) of a single user.Jang et al. (2004) proposed the iris recognition system which can select the good quality data between left and right eye images of same person.

Multiple biometric traits: This category of multiple biometric systems uses multiple sensors for acquiring data pertaining to different traits. That is the evidences of multiple biometric traits are employed for extracting the biometric information of an individual. In multiple biometric traits, a significant improvement in performance is obtained due to the independence of the traits. Brunelli and Falavigna (1995) used face and voice traits in a biometric system for person identification. Sharma and Singh (2017) proposed two uni-modal biometrics, fingerprint and face are used as multi-biometrics. The categories of multibiometric systems are depicted as in Fig. 2 .

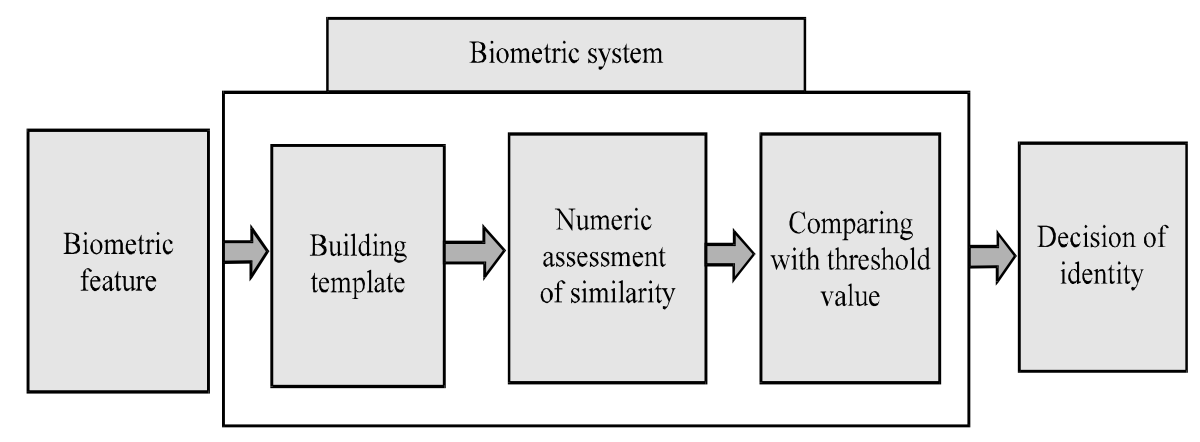

Fig. 1: Diagram of the monomodal biometric system 


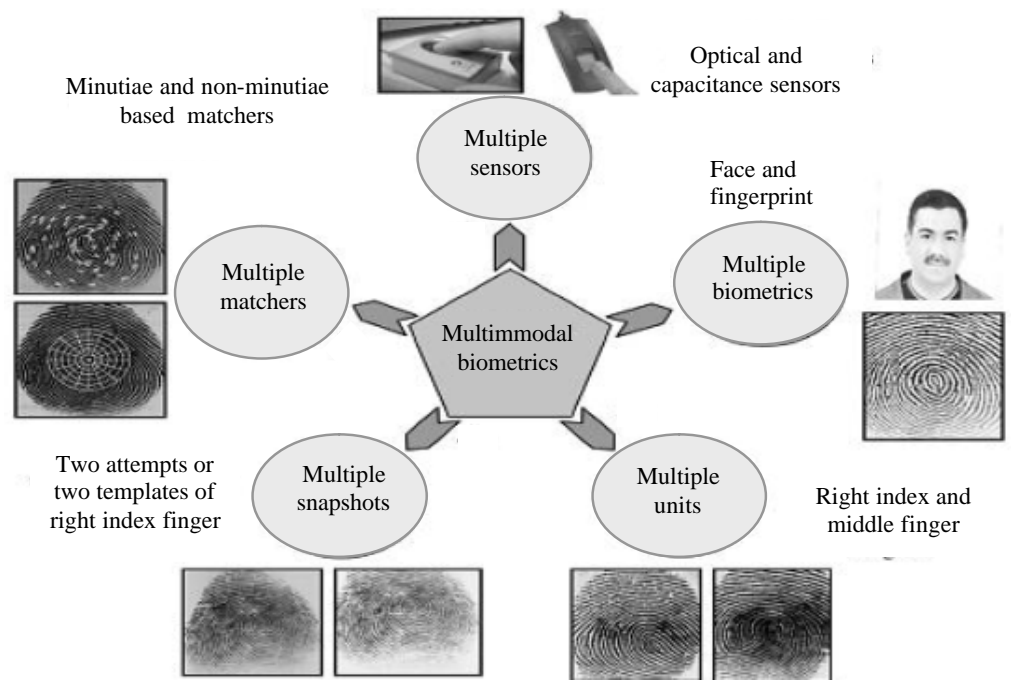

Fig. 2: The different types of multibiometric system

\section{RESULTS AND DISCUSSION}

Implementation: To implementmulti-biometric system, the system has been selected to work with three fingerprints. From biometrics traits, fingerprints are chosen because they are easy to use and give reliable results. The system uses three fingerprint since, a smaller number of biometric features does not give full opportunity to test different strategies for making a decision while greater number slows down the system but does not provide a meaningful difference from the three fingerprints. Figure 3 shows the different stages of implementing the proposed system.

The entire system consists of two main parts: a fingerprint recognition system and a decision making subsystem. The recognition system consists of independent modules, a module for estimating the similarity of two fingerprints and a module for evaluating the quality of the original fingerprint.

Evaluating the quality of fingerprint: To obtain a more accurate evaluation of quality, three algorithms are used to measure the quality of fingerprint according to different criteria.

Algorithm NFIQ: NFIQ (abbreviation of Fingerprint Image Quality) is a standard and widely used algorithm for evaluating the quality of fingerprints. It was developed at the National Institute of Standards and Technology (NIST). When evaluating the quality, it takes into account the direction of the papillary lines, fingerprint contrast and the curvature of the papillary lines. The image is divided into blocks and the quality is evaluated independently in each block. The output of the algorithm is a quality map that contains an evaluation from 1-5 for each block of fingerprint, from it we can get two numerical evaluation- the percentage of poor blocks quality and the average of the entire fingerprint quality. Figure 4 shows fingerprint of high and low quality according to evaluation of NFIQ algorithm. To implement this algorithm, the NFIQ library was used (Anonymous, 2016). Background selection: Also, a background is selected-a part of the image without papillary lines. Figure 5 shows a fingerprint with a large part of the background. This algorithm based on comparing the determined direction in the block and the average of entire fingerprint. The output of the algorithm is a metric that shows the percentage of the background in the entire image.

Evaluating the brightness of fingerprint: Fingerprint with low brightness obtained when a finger which scanned is too wet. This leads to fact that the papillary lines merge together (Fig. 6). Unlike dark, light fingerprint are usually not poor quality and papillary lines are read on them. The evaluation is built by counting those pixels whose brightness is below a certain threshold. The percentage of such pixels to all pixels of the fingerprint determines the quality metric. The evaluation of brightness and background complements the NFIQ quality evaluation, since these parameters are not taken into account in NFIQ.

Evaluating the similarities: The second module of the fingerprint recognition system evaluates the probability with which two fingerprints belong to the same human finger. Before determining the similarity, the quality of the 


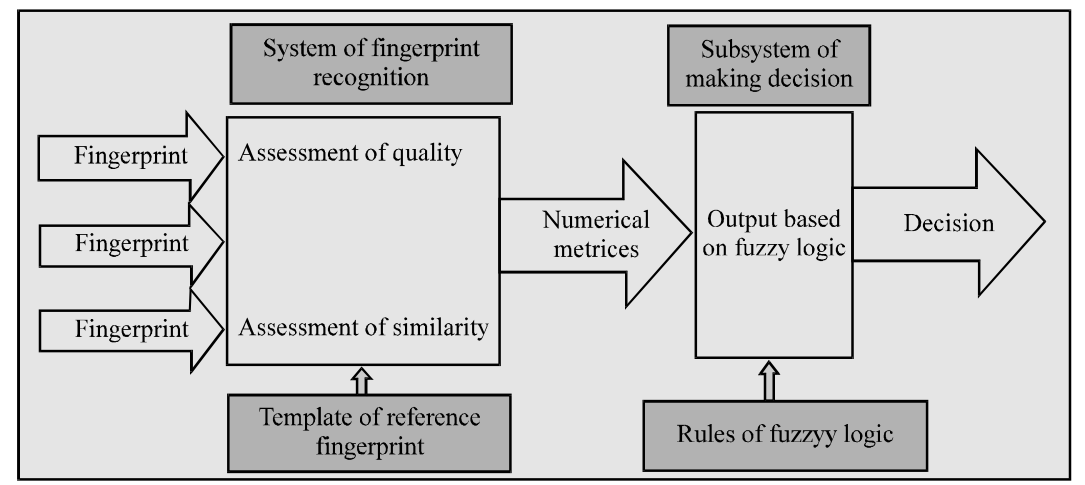

Fig. 3: The architecture of a multimodal biometric system
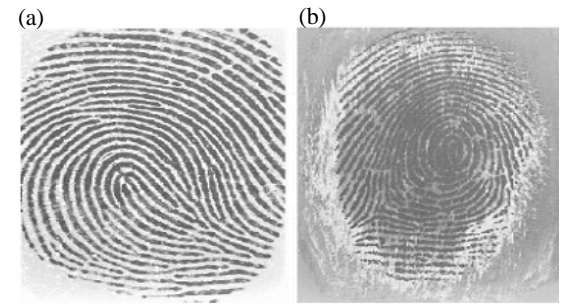

Fig. 4a, b: High and low quality fingerprints according to the NFIQ algorithm

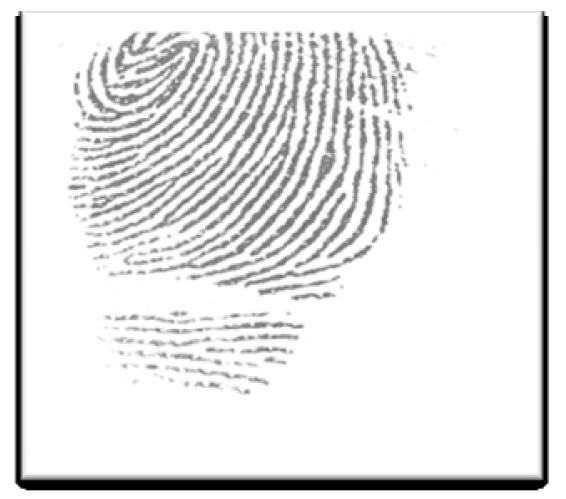

Fig. 5: Fingerprint with a large part of background

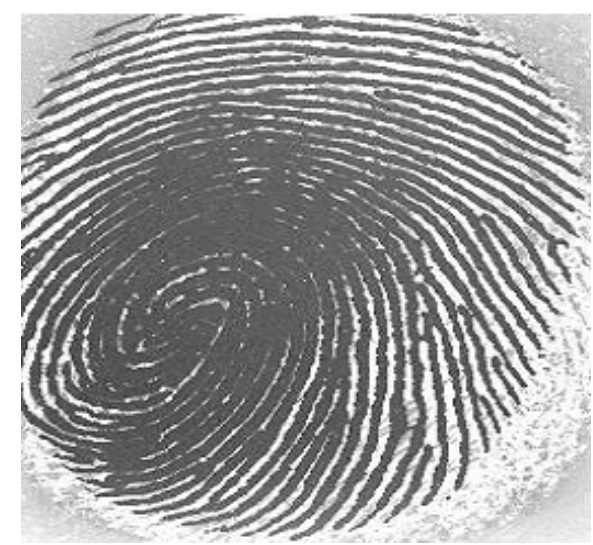

Fig. 6: Fingerprint with merging papillary lines fingerprint image is enhanced for subsequent work. It takes place in three stages, removing noise, binarization and thinning.

To remove noise and enhance the fingerprint ridge pattern, the images were first preprocessed using histogram equalization and fourier transform. The binarization means that the image in grayscale is reduced to black and white. Binarization is done by finding the mean value of each 32-by-32 window and converting the pixel value to 1 if larger than the mean or to 0 if smaller. After converting the image to binary, the thinning algorithm is conducted which reduces the ridge thickness to one pixel wide. Then $\mathrm{H}$ breaks and spikes are removed using morphological operation on thinned image.

After enhancement of the image, Extraction of the minutiae is performed. Minutiae is a special points in the fingerprint on which the papillary line is bifurcates or ends. The mutual arrangement of minutiae and their appearance is unique for each person (Maltoni et al., 2009). Crossing number method used to extract minutiae. This method extracts the ridge endings and bifurcations from the thinned image through examining the neighborhood pixels of $3 \times 3$ window for each ridge pixel.The value of $\mathrm{CN}$ is then calculated, as the half of sum of the differences between pairs of adjacent pixels in the eight-eighborhood.

Then, using these minutiae to build a template-a compact data structure, convenient for comparison. The algorithm used for constructing templates is Minutia Cylinder Code (MCC) (Anonymous, 2018a, b; Conti et al., 2007). MCC algorithm builds "cylinder" for each minutiae which describes position of the nearest minutiae relative to a given one. The measure of the similarity of the two fingerprints is the sum of the hamming distances between the "cylinders".

MCC was developed at the Biometric Systems Laboratory of the Bologna University. This algorithm is 


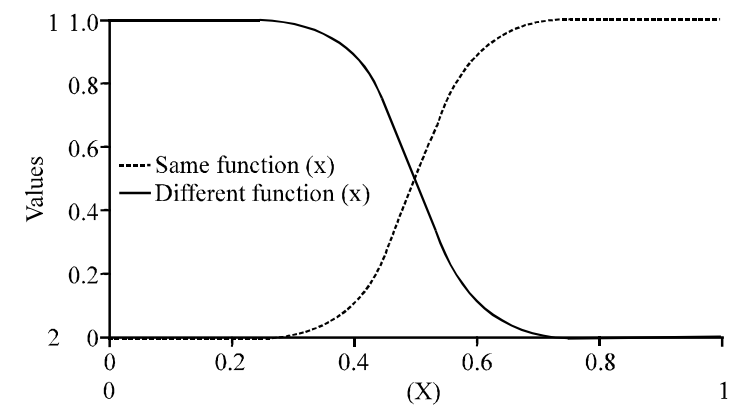

Fig. 7: Membership function of the variable" identity "

resistant to rotations, translation and scaling of the fingerprint. To implement it, the MCC SDK library was used (Anonymous, 2018a, b).

Fuzzy logic decision fusion model: The proposed fuzzy decision making fusion subsystem accepts five numerical metrics for each of the three fingerprints: four quality metrics and one similarity metric with the reference. the fuzzy inference is implemented in three stages; fuzzification, applying the rules and defuzzification. Fuzzification is reduction of numerical metrics to values of linguistic variables. Fuzzification is performed using the corresponding membership functions. The fuzzification and membership functions of the five metrics are shown below.

Fuzzification of similarity: The similarity metric obtained from the MCC algorithm ranges from $0-1$ where 1 means exactly the same fingerprint and values close to 0 are different. This metric is taken as an argument of the membership functions.

When fuzzification, the similarity metrics is the variable identity with the values of same and different. Both these values are fuzzy sets. Their membership functions are shown in Fig. 7. The shift of this graph (Fig. 7) corresponds semantically to the change in the threshold of omparison in the classical recognition systems.

Fuzzification of quality: This process includes fuzzification of the four quality metrics:

Metric of average quality: The average quality metric of the NFIQ algorithm varies from $0-4$ where 0 is a low quality, 4 is the highest quality. The resulting variable is qualitynfiq with the values low, high (Fig. 8).

Metric of poor quality blocks: The metric of the number of poor quality blocks according to the NFIQ algorithm ranges from $0-100$. Poor quality blocks are those with an

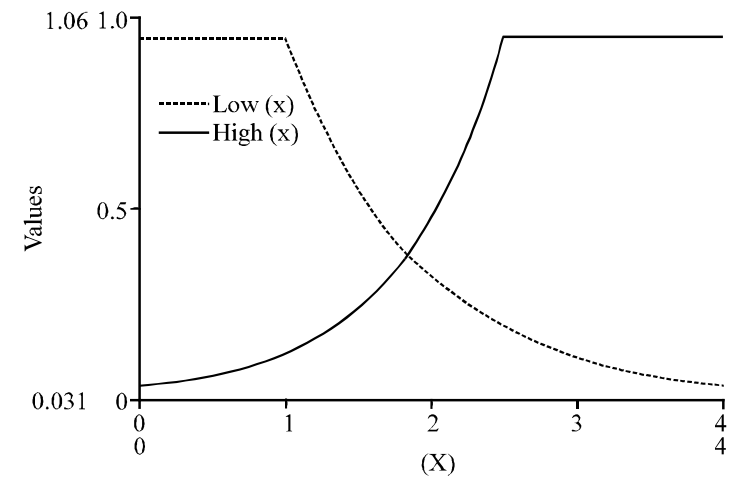

Fig. 8: Membership functions of the variable quality Nfiq

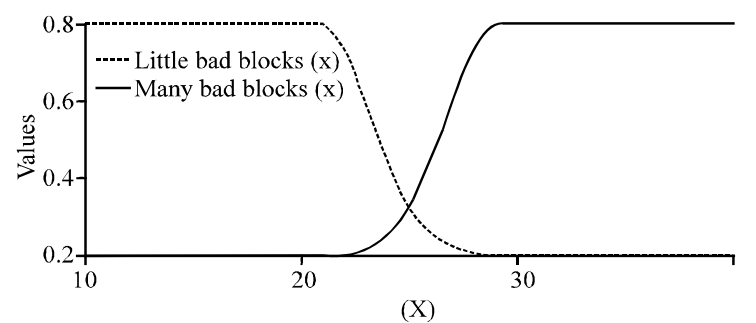

Fig. 9: membership functions of variable low quality blocks

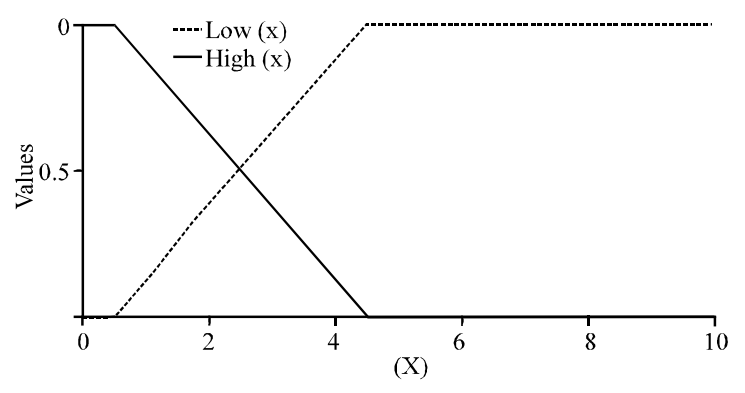

Fig. 10: Membership function of variable brightness

NFIQ score of $0-1$. The resulting variable is low quality block with the values little and many. Here, The membership function is constructed for values from 10 to 40. The values of the function do not change for the values utside the considered range.

Metric of brightness: The brightness metric of the fingerprint also, varies from $0-100$. In this case, values close to 0 characterize the normal fingerprint and large ones are dark. The resulting variable is brightness with high and low. The membership function is constructed for values from $0-10$. The values of the function do not change for the values outside the considered range (Fig. 9 and 10 ). 


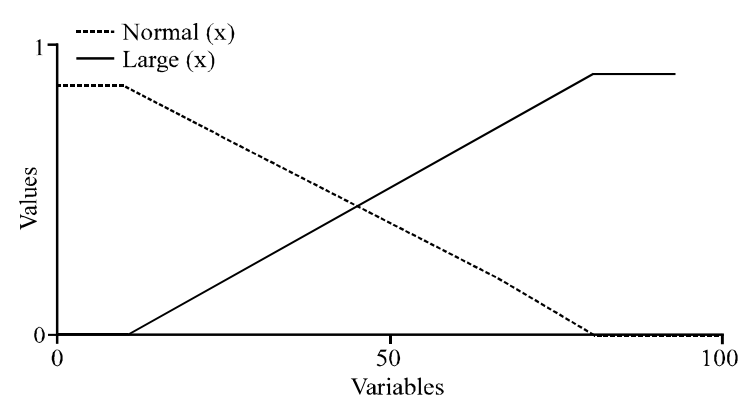

Fig. 11: The membership function of the variable background

Percent metric of background: The percentages metric of background ranges between 0 and 100 . The resulting variable is background with the values large and normal. The form of this membership function was determined empirically (Fig. 11).

Application of rules: The next stage of fuzzy inference is the application of rules. Where, linguistic variables obtained during fuzzification are fed to the input. Since, all the values of the linguistic variable exist simultaneously, the output follows all the rules where it occurs in the premise. The max operation is used to calculate the degree of membership of the premise.

The output of variable "Quality": The quality variable is displayed with the values low, middle and high. It shows the overall quality value for the print. The rules for deriving variable quality were constructed according to the following principle:

- If all the values in the premise are high, then the quality value is high

- If one of the values in the premise is low, then the quality is middle

- If more than one low value, then the quality is low

The similarity output of a single fingerprint: In this part of the output, variables of quality and similarity are used. The rules are constructed according to the following principle:

- If the quality is high, then the answer is the same as the variable of similarity

- If the quality is medium, the answer is with coefficient 0.5 of the variable similarity and with coefficient 0.5 is unknown

- If the quality is low, the answer is unknown

The similarity output of three fingerprints: The last part of the rules applies to the answers from three fingerprints. The principle of building rules can be described as a

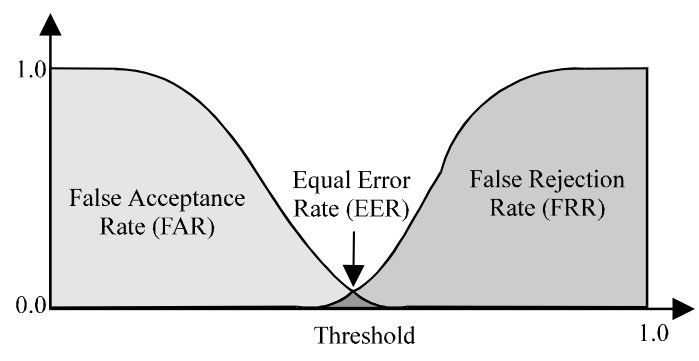

Fig. 12: FAR, FRR and EER errors

\begin{tabular}{lcc}
\multicolumn{3}{l}{ Table 1: ERR of different systems } \\
\hline Variables & Multimodal by majority & Multimodal system with \\
voting system & a response of three out three \\
ERR & $24.18 \%$ & $25.82 \%$ \\
Threshold & 0.53 & 0.53 \\
\hline
\end{tabular}

Table 2: ERR of fuzzy logic decision fusion

\begin{tabular}{llll} 
Method & Level of fusion & Threshold & ERR \\
\hline
\end{tabular}

\begin{tabular}{llll}
\hline Fuzzy fusion & Decision & 0.53 & $13.61 \%$ \\
\hline
\end{tabular}

generalization of voting by a simple majority of three answers. That is if the answers yes, no and Unk (Unknown) are represented as $1,-1$ and 0 , respectively, the overall answer will be obtained by averaging the answers from the three fingerprints and rounding up in the direction of increasing the module. When receiving the general answer, we defuzzify it. The result we get is the answer with the greatest membership degree.

The open database FVC 2000 DB2 set A is used to evaluate the performance of multimodal systems. It consists of 800 fingerprint images, 8 fingerprints per finger. It is worth noting that the FVC 2000 was built to test fingerprint recognition systems and contains many low quality prints. For each finger, the reference finger was considered as the best quality fingerprint of the eight data according to NFIQ.

The performance measure of the system is determined by Equal Error probability (EER) where FAR $=$ FRR (Fig. 12). In an ideal situation, the FAR and FRR graphs do not intersect and the ERR value is zero (Maltoni et al., 2009). The EER of fuzzy fusion based multimodal system is calculated and compared with other two fusion methods (majority voting and a response of three out three ).

Table (1) shows the ERR at threshold value 0.53 for the system with majority voting fusion and the system with fusion of a response of three out three. Table (2) shows the ERR at the same threshold 0.53 for multimodal system with fuzzy logic fusion at decision level.

Experimental results explain an improvement of about 10 and $12 \%$ using the fuzzy logic decision fusion over the fusion by majority voting and with a response of three out of three, respectively . 


\section{CONCLUSION}

The multimodal biometric systems are used to overcome some limitations of a mono- modal systems. The proposed multimodal system based on multi-sample of fingerprints with fuzzy logic fusion at decision level is found to be highly accurate. The research presents an additional improvements for making decision of identity over other systems. Experiments show that the fuzzy logic is practical and efficient solution to these systems influenced by external conditions.

\section{REFERENCES}

Abdolahi, M., M. Mohamadi and M. Jafari, 2013. Multimodal biometric system fusion using fingerprint and iris with fuzzy logic. Intl. J. Soft Comput. Eng., 2: 504-510.

Anonymous, 2016. NIST Biometric Image Software (NBIS). National Institute of Standards and Technology, Gaithersburg, Maryland, USA. https://www.nist.gov/services-resources/software/ nist-biometric-image-software-nbis

Anonymous, 2018. Minutia cylinder-code SDK. Biometric System Laboratory, Bologna, Italy. http://biolab.csr.unibo.it/research.asp?organize $=\mathrm{A}$ ctivities\&select $=\&$ selObj $=82 \&$ pathSubj $=111 \| 8|| 82 \&$ Req $=\& \#$

Anonymous, 2018. Minutia cylinder-code. Biometric System Laboratory, Bologna, Italy. http://biolab. csr.unibo.it/research.asp?organize $=$ Activities\&sele ct $=\&$ selObj $=81 \&$ pathSubj $=111\|8\| 81 \&$ Req $=\&$

Brunelli, R. and D. Falavigna, 1995. Person identification using multiple cues. IEEE Trans. Pattern Anal. Mach. Intell., 17: 955-966.

Conti, V., G. Milici, P. Ribino, F. Sorbello and S. Vitabile, 2007. Fuzzy fusion in multimodal biometric systems. Proceedings of the 11th International Conference on Knowledge-Based and Intelligent Information and Engineering Systems, September 12-14, 2007, Italy, pp: 108-115.

Jain, A., L. Hong and Y. Kulkarni, 1999. A multimodal biometric system using fingerprint, face and speech. Proceedings of the 2nd International Conference on Audio-and Video-based Biometric Person Authentication, March 22-24, 1999, Washington DC., USA., pp: 182-187.

Jang, J., K.R. Park, J. Son and Y. Lee, 2004. Multi-unit iris recognition system by image check algorithm. Proceedings of the 1st International Conference on Biometric Authentication(ICBA' 04), July 15-17, 2004, Springer, Hong Kong, China, ISBN:978-3-540-221463, pp: $450-457$.
Jin, A.T.B., S.A. Samad and A. Hussain, 2003. Theoretic evidence K-nearest neighbourhood classifiers in a bimodal biometric verification system. Proceedings of the 4th International Conference on Audio-and Video-Based Biometric Person Authentication, June 9-11, 2003, Springer, Berlin, Germany, ISBN:978-3-540-40302-9, pp: 778-786.

Kittler, J., M. Hatef, R.P.W. Duin and J. Matas, 1998. On combining classifiers. IEEE Trans. Pattern Anal. Mach. Intell., 20: 226-239.

Lau, C.W., B. Ma, H.M. Meng, Y.S. Moon and Y. Yam, 2004. Fuzzy logic decision fusion in a multimodal biometric system. Proceedings of the 8th International Conference on Spoken Language Processing (ICSLP), October 4-8, 2004, International Convention Center Jeju, Seogwipo, South Korea, pp: 261-264.

Maltoni, D., D. Maio, A.K. Jain and S. Prabhakar, 2009. Handbook of Fingerprint Recognition. 2nd Edn., Springer, Berlin, Germany, ISBN:978-1-84882-253-5, Pages: 494.

Poh, N., S. Bengio and J. Korczak, 2002. A multisample multi-source model for biometric authentication. Proceeding of the 12th International Workshop on Neural Networks for Signal Processing, November 7, 2002, IEEE., pp: 375384 .

Prabhakar, S. and A.K. Jain, 2002. Decision-level fusion in fingerprint verification. Pattern Recognit., 35: 861-874.

Ross, A. and A.K. Jain, 2004. Multimodal biometrics: An overview. Proceedings of the 12th European International Conference on Signal Processing, September 6-10, 2004, IEEE, Vienna, Austria, ISBN:978-320-0001-65-7, pp: 1221-1224.

Ross, A., A. Jain and J. Reisman, 2003. A hybrid fingerprint matcher. Pattern Recognit., 36: 1661-1673.

Sharma, P. and K. Singh, 2017. Multimodal biometric system fusion using fingerprint and face with fuzzy logic. Intl. J. Adv. Res. Comput. Sci. Software Eng., 7 : 482-489.

Vasuhi, S., V. Vaidehi, N.N. Babu and T.M. Treesa, 2010. An efficient multi-modal biometric person authentication system using fuzzy logic. Proceedings of the 2nd International Conference on Advanced Computing (IcoAC'10), December 14-16, 2010, IEEE, Chennai, India, ISBN:978-1-61284-261-5, pp: 74-81. 